\title{
LA DETECCIÓN DE LOS CASOS DE ABUSO SEXUAL INFANTIL DESDE LOS SERVICIOS SOCIALES GENERALES: PRINCIPALES DIFICULTADES Y ALGUNAS SUGERENCIAS PARA SU SOLUCIÓN.
}

\author{
ENCARNACIÓN GUILLÉN SÁDABA \\ $M^{a}$ CARMEN ALEMÁN BRACHO \\ ANDRÉS ARIAS ASTRAY \\ FERNADO DE LUCAS Y MURILLO DE LA CUEVA \\ DARIO PEREZ MADERA \\ E.U. Trabajo Social. Universidad Complutense. Madrid.
}

INTRODUCCIÓN.

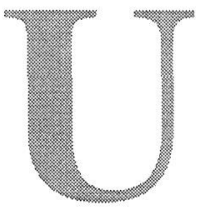

na de las condiciones necesarias, aunque no suficientes, para luchar de forma efectiva contra los casos de abuso sexual infantil (ASI de aquí en adelante) consiste en detectarlos de forma adecuada (DePanfilis y Salus, 1992). Los Servicios Sociales Generales tienen en este sentido una importante responsabilidad, dado que como todos sabemos representan la respuesta más cercana a los ciudadanos ante cualquier demanda o necesidad que estos puedan presentar (Casado y Guillén, 2001). El problema estriba en que la identificación de los ASI no suele resultar en casi ningún caso una tarea sencilla. Las dificultades que implica son múltiples y de diferente orden, y las soluciones a las mismas, dado los recursos y el grado de conocimientos y habilidades que requieren, no siempre son fáciles de implementar desde el primer nivel de atención.

Por otra parte, es necesario tener en cuenta las consecuencias, no siempre evidentes, que se derivan de una detección incorrecta de un caso ASI. Los falsos negativos ${ }^{1}$ permiten que el maltrato o abuso continúen, niegan a la víctima la asistencia y protección adecuadas causando graves daños psicológicos a los menores y a quie-

1 Un falso negativo supone concluir que no se han dado abusos sexuales cuando realmente sí han ocurrido, y un falso negativo consiste en que se considera que tales abusos han sucedido cuando en realidad no es así. 
nes los cuidan y defienden. Los falsos positivos pueden acarrear consecuencias muy perniciosas para personas que son inocentes de lo que se les acusa (separación innecesaria de los hijos, penas de cárcel, ruptura de la familia, pérdida de la reputación, pérdida del trabajo, etc.) $\mathrm{y}$, en algunas ocasiones, para los propios menores al crear en ellos una falsa identidad como víctimas (O’Donohue y Fanetti, 1996; Denninson, 1996; Jones, 2001).

Pasemos, pues, a analizar brevemente cuáles son algunas de las dificultades que pueden impedir o limitar la detección correcta de los casos de ASI desde los Servicios Sociales Generales y cuáles las medidas que se pueden implementar para solventarlas o, al menos, minimizarlas.

Para facilitar nuestra exposición vamos a distinguir dos grandes tipos de dificultades: Las primeras, a las que denominaremos "dificultades de orden general", estarían relacionadas con factores que transcienden a los protagonistas principales de los casos de ASI y su detección. Tendrían que ver aspectos relacionados con la estructura social e institucional, así como con su dinámica. Las segundas, a las que daremos el nombre de "dificultades especificas", vendrían dadas por las características personales y contextuales de las víctimas, de los maltratadores e incluso de los profesionales que intervienen directamente en los casos de ASI y su detección.

\section{DIFICULTADES DE ORDEN GENERAL.}

El tipo de dificultades que se pueden incluir en este primer grupo serían, en muchos casos, similares a las que impiden la correcta detección, por parte de los diferentes sistemas de protección social, de afectados por otros problemas sociales que también se caracterizan por una cierta invisibilidad. Dejando al margen aquéllas que tienen que ver con la limitación de medios que siempre caracteriza las actuaciones de los Servicios Sociales (pues su solución, aunque difícil, es evidente), en el caso de los ASI, podemos subrayar, en principio, las siguientes (como se podrá observar, muchas de ellas están íntimamente relacionadas):

1. Aunque se han realizado importantes avances en este sentido, todavía no se cuenta con una definición universalmente aceptada de lo que constituye el ASI (Russell, 1988; Zastrow, 1999; Echeburúa y Guerricaechevarría, 2000).

2. Debido a ello, aunque también por otras razones ${ }^{2}$, el rango en el que

2 Sobre todo metodológicas. Para una revisión de este tema pueden consultarse, entre otros, los trabajos de Bradley y Lindsay (1987), Widom (1988), Melton y Flood (1994). 
varían las estimaciones sobre tasas de incidencia y prevalencia de ASI disponibles es demasiado amplio, ${ }^{3}$ aunque se piensa que son, en general, menores a las reales (Finkelhor, 1986; Russell, 1988, Inglés, 1995; López, Hernández y Carpintero, 1995; López, 1997; McDonald, 1998). ${ }^{4}$

3. Íntimamente relacionado con lo anterior, y a pesar de la alarma que despiertan en la población las noticias relacionadas con los ASI, todavía no existe la suficiente conciencia social sobre su extensión, gravedad y consecuencias. Del mismo modo, aún se comparten muchos tópicos e ideas inadecuadas sobre los mismos (López, 1997), algunos de los cuales contribuyen a difuminar la responsabilidad de los ciudadanos a la hora de detectarlos y a que todavía sean muy pocos los casos que se denuncian.

Entre estos últimos podemos indicar, a modo de ejemplo, que se tiende a pensar que los abusos sexuales son poco frecuentes, cuando en España se estima que el $23 \%$ de las mujeres y el $15 \%$ de los hombres han sido víctimas de algún tipo de abuso sexual durante su infancia; que sólo afectan a determinadas clases sociales cuando de hecho no es cierto; que si de verdad le ocurriera a alguien cercano nos daríamos cuenta, cuando se estima que en el momento de su ocurrencia sólo se llegan a detectar un $2 \%$ de los casos, etc. (véase Ib; cfr. Echeburúa y Guerricaechevarría, 2000, p. 12).

4. Algunos de los dispositivos existentes para hacer frente a los ASI son poco conocidos por la población. Tal parece ser el caso de los Servicios Sociales Generales, y de forma particular de los Servicios Sociales Generales, que no se presentan ante los ciudadanos como dispositivos en los que se tratan éste y otro tipo de situaciones para los que tienen competencia.

5. Por otra parte, es preciso apuntar que, a pesar de que se están dando pasos en esta dirección ${ }^{5}$, desde los Servicios Sociales toda-

Biere (1992), McGee, Wolfe, Yuen, Wilson y Carnochan (1995) y Goldman y Dayachi (2000).

3 Diferentes estudios internacionales han mostrado que entre el $6 \%$ y el $62 \%$ de las mujeres y el $3 \%$ y el $30 \%$ de los hombres han sufrido una experiencia de abuso sexual antes de los dieciocho años. Las tasas de incidencia más altas pertenencen a estudios con criterios menos estrictos para definir el abuso sexual, y las más bajas a estudios que atienden a actos de violencia sexual (McDonald, 1998).

4 Como afirman López, Hernández y Carpintero (1995), los datos con los que se cuenta sobre ASI representan más un "índice del nivel de funcionamiento de los profesionales y de los Servicios Sociales de un país, que del número real de abusos sexuales cometidos".

5 Podemos citar, entre otros, el ejemplo que en este sentido suponen los trabajos realizados en Cataluña (en concreto en Girona) y Canarias, representados, respectivamente, en el "Protocolo básico de actuaciones en abusos sexuales y otros malos tratos a menores en la demarcación de Girona" y en el "Protocolo de facilitación de detección de malos tratos a Mujeres y Menores". 
vía no se cuenta con protocolos de atención específicos y, lo que es más importante, homogeneizados para la detección de casos de ASI. Tampoco poseemos sistemas uniformes de registro de casos, perdurables en el tiempo y sistemáticos, para todo el territorio nacional. Algo que sin duda redundaría no sólo en una protección más adecuada de estas víctimas, sino en una mejor estimación de la magnitud del problema con el que nos enfrentamos.

6. Finalmente, hay que considerar las dificultades provocadas por la excesiva compartimentalización y falta de coordinación entre los recursos generales y especializados, y entre los distintos sistemas de protección social. De modo muy especial, aquellas que se derivan de la pérdida de usuarios generada durante los procesos de derivación.

Las limitaciones de tiempo y espacio nos impiden comentar con mayor detalle éstas y algunas otras dificultades generales que se podrían apuntar. Sin embargo, antes de pasar a comentar las de tipo específico, es necesario que realicemos, al menos, un par de sugerencias sobre el modo en el se pueden superar o minimizar las que acabamos de referir.

En primer lugar, sería necesario establecer, por parte de las autoridades competentes (e.g. Ministerios de Salud, Trabajo y Servicios Sociales, Educación, Justicia e Interior), comisiones de coordinación interterritorial e intersectorial en las que estuviesen representados, al menos, los ministerios que se acaban de referir, todas y cada una de las CCAA, la Federación Española de Municipios y Provincias, así como las principales ONGs implicadas en la protección de la infancia. Como objetivos prioritarios sugerimos, con independencia de otros muchos que se puedan plantear, acordar una definición de trabajo consensuada sobre los ASI; establecer una metodología homogénea sobre su registro; crear un banco de datos centralizado de los casos estudiados y detectados y abierto a los investigadores que se ocupan de estos temas; establecer un modelo único de atención para cada uno de los sistemas de protección social implicados en el tema, particularizando, cuando sea el caso, los diferentes protocolos aplicables en cada uno de los niveles de atención; elaborar e implementar programas de comunicación para incrementar el conocimiento de la población sobre los ASI y sobre los dispositivos de atención existentes para hacer frente a los mismos.

En segundo lugar, sería conveniente fomentar la investigación básica y aplicada sobre los ASI en nuestro país, así como la colaboración e integración de los profesionales que trabajan directamente con la infancia y los equipos de investigación. En este sentido es necesario subrayar que, si bien es cierto que ya se cuenta con unos cuantos 
grupos de investigación consolidados (e.g. López y cols.), en muchos casos nos manejamos con supuestos derivados de investigaciones realizadas en otros contextos culturales que, tal vez, no sean directamente aplicables en nuestro medio.

\section{DIFICULTADES ESPECÍFICAS.}

En este caso tratamos alguna de las dificultades que limitan la identificación de los casos de ASI y que tienen que ver directamente con variables asociadas con la situación y características de la víctima, del agresor y de los profesionales que trabajan en este campo. En el caso de estos últimos nos referiremos especialmente a los que trabajan en los Servicios Sociales Generales y, en concreto, a los trabajadores sociales.

En lo que respecta a la situación y características de los menores víctimas de ASI, es necesario tener en cuenta una serie de aspectos que, como rápidamente se observará, dificultan en extremo la detección de estos casos. Sin carácter exhaustivo se pueden apuntar los siguientes:

1. En la mayoría de las ocasiones las únicas pruebas disponibles para la detección de los ASI son las declaraciones de los menores, pues las más de las veces las evaluaciones médicas realizadas suelen mostrarse dentro de los límites de la normalidad (Caballero, 1999; Lahoti, McClain, Girardet, McNeese y Cheung, 2001).

En concreto, DeJong (1992) ha estimado que sólo se constatan indicadores físicos del ASI en un $10 \%$ de los casos. Por otra parte hay que apuntar que existen una serie de indicadores comportamentales y emocionales que pueden sugerir la existencia de abusos sexuales, pero que en casi ningún caso son determinantes, pues pueden ser consecuencia de otras muchas circunstancias (López y del Campo, 1997; World Health Organization. Fact Sheet $N^{\circ} 150$ ).

2. Si bien es cierto que se ha exagerado más de lo debido el grado de sugestibilidad de los menores a la hora de informar sobre episodios de abuso sexual (McDonald, 1998; Lyon, en prensa), y por lo tanto el riesgo de cometer falsos positivos, sí es verdad que tal problema existe (Ceci y Bruck, 1993; Lewis, Wilkins, Baker y Woobey, 1995; Bruck y Ceci, 1999; Cantón y Cortés, 2000a; Jones, 2001). Su solución pasa, sin duda, por una adecuada entrevista del menor, que incluya estrategias para adaptarse a sus características (Koriat, Godsmith, Schneider, Nakash-Dura, 2001).

3. Otra cuestión que no se debe pasar por alto es el bajo porcentaje de menores que confiesan estar sufriendo abusos sexuales (Finkelhor, 
1986; McDonald, 1998; Paine y Hansen, 2002), entre otras razones, por la culpabilidad y vergüenza que sienten y por el miedo a las consecuencias que tal declaración pueda tener para sí mismos, sus seres queridos o los agresores (Lyon, 2001).

4. Relacionado con lo anterior, es imprescindible recordar que un alto porcentaje de los ASI son perpetrados por personas que conviven con el menor, con las limitaciones que ello impone para que éste termine realizando una confesión. Hay que advertir, que es tristemente frecuente el incesto, sea éste cometido por los padres o por los hermanos mayores.

Según afirman Echeburúa y Guerricaechevarría (2000, pp. 13-14, citando a McCarthy, 1992) hay un mayor número de niñas en el abuso intrafamiliar (incesto), con una edad de inicio anterior (7-8 años), y un mayor número de niños en el extrafamilar (pedofilia), con una edad de inicio posterior (11-12 años). La mayoría de las investigaciones coinciden en que el agresor suele ser conocido por la víctima... No más del $20 \%$ de los casos denunciados de incesto hacen referencia a los contactos padre-hija... El incesto entre padrastro e hija da cuenta del 15\%-20\% de los casos. El 65\% restante implica a hermanos, tíos, hermanastros, abuelos y novios que viven en el mismo hogar".

5. Por último, es preciso tener en cuenta, como afirman Cortés y Cantón (2000, p. 37), que en algunas ocasiones la reacción de las familias (especialmente de las madres) ante la revelación de los abusos, "no siempre es de apoyo, sino que algunas se niegan a creer al niño, reaccionando incluso de manera colérica y rechazándolo". Asimismo, se ha constatado que entre las razones principales por las cuales los padres se niegan a denunciar los ASI (López y Del Campo, 1997), se encuentran el temor a las consecuencias que pueda generar la publicidad del caso, especialmente cuando el agresor es un familiar; el sentimiento que la denuncia y el proceso subsiguiente no reparará los daños sufridos; el desconocimiento de los procesos a seguir y la poca confianza en y el temor $a$ las consecuencias de un proceso judicial.

En relación con las características de los agresores que van limitar las probabilidades de detección de los ASI, además de lo que acabamos de referir, es conveniente recordar, entre otros aspectos, los siguientes:

1. No existe un prototipo de agresor. Si bien es cierto que algunos autores (Id) han intentado ofrecer su "retrato robot", indicando, entre otros aspectos, que suelen ser mayoritariamente varones con apariencia de normalidad, no pedófilos pero con dificultades para rela- 
cionarse con sus iguales.

2. En un $90 \%$ de los casos no suele utilizarse la violencia para abusar de las víctimas, ni para conseguir su silencio. Su principal forma de defenderse, una vez que existe la sospecha de abuso, consiste en negar los hechos, manipular directa o indirectamente al menor y al resto de la familia (en concreto dividiendo lealtades), y presentarse como víctima de las mentiras o imaginación del niño (Id, Zastrow, 1999, p. 556).

Pasando a comentar, ya para finalizar, las dificultades derivadas de la situación y características de los profesionales que en los Servicios Sociales Generales se tienen que encargar de la detección de los casos de ASI, podemos indicar lo siguiente.

1. Las funciones que desempeñan los profesionales, unidas a la sobrecarga de trabajo que suelen soportar, hace que éstos se enfrenten con una paradoja difícil de solventar: tienen que resolver, con vocación generalista, casos que requieren, ya en su detección, una intervención altamente especializada. Una tesitura que es necesario solventar, entre otras cosas, porque los profesionales del primer nivel de atención desarrollan su actividad en uno de los contextos privilegiados para conseguir tal detección.

2. En concreto, requieren y no suelen disponer de la suficiente formación sobre los ASI en general y sobre las variables que pueden afectar a su detección en particular. Una formación que pasaría, entre otros aspectos, por habilidades para relacionarse con y entrevistar $a$ menores. De la importancia de esta afirmación dan fe los casos de falsos abusos sexuales infantiles creados por el alarmismo y la inadecuada práctica profesional de algunos individuos tristemente conocidos. ${ }^{6}$

Aunque no referido a España, un dato significativo en este sentido nos lo ofrecen Wood, McClure y Kimberly (1996) tras analizar las características de las entrevistas realizadas por profesionales pertenecientes a entidades encargadas de su protección y constatar que en un amplio número de casos no se ajustaban a las pautas recomendables para su correcta realización.

3. Por otra parte, la creciente tendencia a la burocratización de las intervenciones que se realizan desde el primer nivel de atención, pueden impedir la creación de la atmósfera necesaria para favorecer la revelación de un menor que ha sufrido un abuso sexual. De este modo, sería difícil llegar a adoptar las pautas recomendadas

6 Por ejemplo, el caso de Margaret Michels, profesora del jardín de Infancia "We Care Nursey" o el muy reciente del bebé que apareció muerto en la piscina. 
por López y del Campo (1997) para contribuir a este proceso: propiciar la confianza de los niños y escucharles, demostrarle al niño que se le cree, eliminar cualquier componente de culpabilidad, hacer que se enorgullezca de haber dado ese primer paso, ofrecerles la seguridad de que nada les va a suceder, que el abuso no se volverá a repetir y que no debe temer ninguna represalia, etc.

4. Finalmente, existen otra serie de aspectos igualmente importantes para la adecuada detección de los ASI. Tienen que ver con la experiencia personal del profesional y se vinculan directamente con la neutralidad que es necesaria para llevar este tipo de casos. Así, el hecho de ser madre o padre, o el haber sido víctima de abusos sexuales, por ejemplo, aunque en un principio no tienen que impedir ni disminuir la capacidad para conducir de modo adecuado la intervención en este tipo de situaciones, deben ser sometidos a supervisión. En la misma línea, hay que tener el cuenta la incidencia del Síndrome de "burnout" en los profesionales que, de modo frecuente, se han de enfrentar a este tipo de casos (North Carolina's Child Welfare Workers, 2000).

Las soluciones a este segundo grupo de dificultades para la detección de los ASI, además de las que ya se han referido más arriba, ${ }^{7}$ pasan principalmente por la formación y preparación, tanto personal como técnica, de los profesionales de los Servicios Sociales Generales. Una capacitación, por otra parte, que ha de iniciarse durante los Estudios Universitarios (que han de inculir en el curriculum contenidos relativos a los malos tratos en general y a los ASI en particular), y mantenerse en el tiempo, mediante cursos de formación continua ofrecidos por las diferentes entidades sociales en las que desempeñen su labor.

A pesar de que nos repitamos, para este último tipo de dificutades también será de especial relevancia el desarrollo de materiales formativos tanto para los profesionales como para el resto de la comunidad. En el primer caso estamos haciendo referencia, entre otros elementos, a protocolos de atención y evaluación que orienten al profesional de los Servicios Sociales (Cantón, 2000; Cantón y Cortés, 2000b). En ambos casos habrá de incluirse información no sólo sobre lo que se debe hacer ante la sospecha de un ASI, sino también sobre lo que es conveniente que no haga. En definitiva, hacer conscientes tanto al

7 De especial relevancia en este sentido es el desarrollo de materiales formativos $y$, en concreto, de protocolos que orienten al profesional de los Servicios Sociales no sólo sobre lo que debe hacer ante la sospecha de un abuso sexual infantil, sino también sobre lo que es conveniente que no haga. En definitiva, que el profesional sea consciente de los efectos iatrogénicos que puede generar su participación en un caso de A.SI sin estar lo suficientemente capacitado. 
profesional como a los miembros de la comunidad de los efectos iatrogénicos que puede generar su participación en un caso de ASI sin estar lo suficientemente preparado.

Finalmente, otro aspecto de importancia capital está relacionado con el apoyo que desde los Servicios Sociales se puede ofrecer a los familiares de las víctimas de ASI, especialmente a las madres, tratando, siempre que sea posible, que las actuaciones del sistema no contribuyan a añadir nuevos problemas a los que se pretenden solventar (Cortés y Cantón, 2000).

\section{BIBLIOGRAFÍA}

BRIERE, J.: "Methodological issues in the study of sexual abuse effects". Journal of Consulting and Clinical Psychology, 1992, 60, 196-203.

BRADLEY, E.J. \& LINDSAY, R.C.: "Methodological and ethical issues in child abuse research". Journal of Family Violence, 1987, 2 , 239-253.

BRUCK, M. y CESSI, S.J.: "The suggestibility of children memory”. Annual Review of Psychology, 1999, 50, 419-439.

CABALLERO, M.A.: "Valoración inicial de casos de abuso sexual infantil". Trabajo Social y Salud, 1999, 33, 303-341.

CANTÓN, J.C. y CORTÉS, M.R.: “La sugestibilidad de los niños”, en J. Cantón y M.R. Cortés (Eds.) Guía para la evaluación del abuso sexual infantil. Madrid: Pirámide, 2000a, 85-122.

CANTÓN, J.C. y CORTÉS, M.R.: "Guía para una entrevista de investigacion". en J. Cantón y M.R. Cortés (eds.) Guía para la evaluación del abuso sexual infantil, Pirámide, Madrid, 2000b, 147-179.

CANTÓN, J.C.: "Guías y protocolos publicados para la realización de la entrevista de investigación", en J. Cantón y M.R. Cortés (eds.), Guía para la evaluación del abuso sexual infantil, Pirámide, Madrid, 2000,123-146.

CASADO, D. y GUILLÉN, E.: Manual de Servicios Sociales, CCS, Madrid. 2001.

CECI, S.J. y BRUCK, M.: "Suggestibility of the child witness: a historical review and synsthesis", Psychological Bulletin, 1993, 113, 403-439.

CORTÉS, M. R. y CANTÓN, J.: "El abuso sexual infantil: un grave problema social”, en J. Cantón y M.R. Cortés (eds.), Guia para la evaluación del abuso sexual infantil, Pirámide, Madrid, 2000, 13-52.

DEJONG, A.R.: "Medical detection and effects of the sexual abuse of children", en W. O'Donohue y J. Geer (Eds.), The sexual abuse of children: clinical issues, Earlbaum, Hillsdale, NJ, 1992, 71-99.

DENNINSON, R.D.: "Findings from research on children's suggestibility and imprlications for conducting child interviews", Child Matreatment, 1996, 
$1,105-131$.

DEPANFILIS, D. y SALUS, M.K.: Child protective Services: a guide for caseworkers. US Department of Health and Human Services. Adminisration for Children and Familises. Administration on Childre, Youth and Families. National Center on Child Abuse and Neglect, 1992.

ECHEBURÚA, E. y GUERRICAECHEVARRÍA, C.: A buso sexual en la infancia: víctimas y agresores. Un enfoque clínico, Ariel, Barcelona, 2000.

FINKELHOR, D.: A sourcebook on child sexual abuse, Sage, Beverly Hills, CA, 1986.

GOLDMAN, J.D.G. y PADYACHI, U.K.: "Some methodological problems in estimating incidence and prevalence in child sexual abuse research", Journal of Sex Research, 2000, 34, 305-314.

JONES, D.P.H.: "Editorial - false positives in the field of child maltreatment", Child Abuse \& Neglect, 2001, 25, 1395-1396.

KORIAT, A.; GODSMITH, M.; SCHNEIDER, W; NAKASH-DURA, M.: "The credibility of children's testimony: can children control the accuracy of their memory reports", Journal of Experimental Child Psychology, 2001, $79,405-437$.

LAHOTI, S.L.; MCCLAIN, N.; GIRARDET, R.; MCNEESE, M. y CHEUNG, K.: "Evaluating the child for sexual abuse". American Family Physician, 2001, 63, 883-892.

LEWIS, CH.; WILKINS, R.; BAKER, L. y WOOBEY, A.: «"Is this man your daddy" Sugestibility in children's eyewitness identification of a family member». Child Abuse and Neglect, 1995, 19, 739-744.

LÓPEZ, F.: "Abuso sexual: un problema desconocido", en J. Casado, J. A. Díaz y C. Martínez (Eds.), Niños maltratados, Díaz Santos, Madrid, 1997.

LÓPEZ, F. y DEL CAMPO, A.: Prevención de abusos sexuales a menores. Guía para los educadores, Amaru Ediciones, Salamanca, 1997.

LÓPEZ, F.; HERNÁNDEZ, A. y CARPINTERO, E.: "Los abusos sexuales de menores: concepto, prevalencia y efectos", Infancia y Aprendizaje, 1995, 71,77-98.

LYON, T.D.: "Scientific support for expert testimony on child sexual abuse accommodation", en J. Conte (Ed.), The knowns and unknowns of child sexual abuse, Sage, Thousand Oaks, 2001.

LYON, T.D.: "Let's no exaggerate the suggestibility of children", The Judges Journal, en prensa.

MELTON, G. B., \& FLOOD, M. F.: "Research policy and child maltreatment: Developing the scientific foundation for effective protection of children", Child Abuse \& Neglect, 1994, 18, 1-28.

MCDONALD, M.: "The myth of epidemic false allegations of sexual abuse in divorce cases", Court Review, 1988, 35, 12-26.

MCGEE, R.A., WOLFE, D.A., YUEN, S.A., WILSON, S.K., \& CARNOCHAN, 
J.: "The measurement of maltreatment: A comparison of approaches", Child Abuse \& Neglect, 1995, 19, 233-249.

O'DONOHUE, W. y FANETTI, M.: "Assessing the occurrence of child sexual abuse: an information processing, hipothesis testing approach", Aggression and Violent Behavior, 1996, 3, 269-281.

PAINE, M.L. y HANSEN, D.J.: "Factors influencing children to self-disclose sexual abuse", Clinical Psychology Review, 2002, 22, 271-295

RUSSELL, S.J.: "The incidence and Prevalence of intrafamiliar and extrafamilar abuse of female children", en L. E. A. Walker (Ed.), Handbook on Sexual abuse of Children, Springer, Nueva York, 1988, 19-36.

WIDOM, C.: "Sampling biases and implications for child abuse research", American Journal of Orthopsychiatry, 1988, 58, 260-270.

WOOD, J.M. Y MCCLURE K.A.: "Suggestions for improving interviews in child protection agecies", Child Maltreatmen, 1996, 1, 223-231.

WORLD HEALTH ORGANIZATION: "Fact Sheet $N^{\circ} 150$. Child Abuse and Neglect", http://www.who.org/inf/fs/fact150.html, 2002, 10 de enero.

ZASTROW, CH.H.: The practice of Social Work. $\sigma^{a}$ ed., Brooks/Cole, Pacific Grove, 1999. 